\title{
Dark Matter and HI in Ultra-Diffuse Galaxy UGC 2162
}

\author{
Chandreyee Sengupta, ${ }^{1 \star}$ T. C. Scott ${ }^{2}$, Aeree Chung ${ }^{3}$, O. Ivy Wong ${ }^{4}$ \\ ${ }^{1}$ Purple Mountain Observatory, No.8 Yuanhua Road, Qixia District, Nanjing 210034, China \\ ${ }^{2}$ Institute of Astrophysics and Space Sciences (IA), Rua das Estrelas, 4150-762 Porto, Portugal \\ ${ }^{3}$ Department of Astronomy, Yonsei University, 50 Yonsei-ro, Seodaemun-gu, Seoul, Republic of Korea \\ ${ }^{4}$ International Centre for Radio Astronomy Research (ICRAR), University of Western Australia, 35 Stirling Highway, WA 6009, Australia
}

\begin{abstract}
Our GMRT H I observations of the ultra diffuse galaxy (UDG) UGC 2162, projected 300 kpc from the centre of the M 77 group, reveal it to a have an extended $\mathrm{H}$ I disk $\left(\mathrm{R}_{H I / R_{25}} \sim 3.3\right)$ with a moderate rotational velocity $\left(\mathrm{V}_{\text {rot }} \sim 31 \mathrm{~km} \mathrm{~s}^{-1}\right)$. This $\mathrm{V}_{\text {rot }}$ is in line with that of dwarf galaxies with similar $\mathrm{H}_{\mathrm{I}}$ mass. We estimate an $\mathrm{M}_{d y n}$ of $\sim 1.14 \times 10^{9} \mathrm{M}_{\odot}$ within the galaxy's $\mathrm{R}_{H I} \sim 5.2$ $\mathrm{kpc}$. Additionally, our estimates of $\mathrm{M}_{200}$ for the galaxy from NFW models are in the range of 5.0 to $8.8 \times 10^{10} \mathrm{M}_{\odot}$. Comparing UGC 2162 to samples of UDGs with H I detections show it to have amongst the smallest $\mathrm{R}_{e}$ with its $\mathrm{MH} / \mathrm{M}_{*}$ being distinctly higher and $\mathrm{g}-\mathrm{i}$ colour slightly bluer than typical values in those samples. We also compared $\mathrm{H}_{\mathrm{I}}$ and dark matter (DM) halo properties of UGC 2162 with dwarf galaxies in the LITTLE THINGS sample and find its DM halo mass and profile are within the range expected for a dwarf galaxy. While we were unable to to determine the origin of the galaxy's present day optical form from our study, its normal $\mathrm{H}$ I rotation velocity in relation to its $\mathrm{H}$ I mass, $\mathrm{H}_{\mathrm{I}}$ morphology, environment and dwarf mass DM halo ruled out some of the proposed ultra diffuse galaxy formation scenarios for this galaxy.
\end{abstract}

Key words: galaxies: ISM - galaxies: interactions - galaxies: kinematics and dynamics galaxies: individual: UGC 2162 - radio lines: galaxies

\section{INTRODUCTION}

Since the discovery of forty seven ultra diffuse galaxies (UDG) in the Coma cluster by van Dokkum et al. (2015a), there have been several further reports of many more UDGs, mostly in galaxy clusters (e.g., Yagi et al. 2016; Wittmann et al. 2017; Toloba et al. 2018). UDGs have $M_{*}$ of $10^{7}$ to $10^{8} \mathrm{M}_{\odot}$, a central $\mathrm{g}-$ band surface brightness $\left(\mu_{g}\right)$ of $>24$ mag $\operatorname{arcsec}^{-2}$ and an effective radius ${ }^{1}\left(R_{e}\right)$ of $\sim 1.5-5 \mathrm{kpc}$. UDGs are found in a range of environments from clusters and groups to in isolation, with their relative abundance increasing from the field to the centres of massive galaxy clusters (van der Burg et al. 2017). This implies a significant role for environment in the evolution at least some of them (Carleton et al. 2019). UDGs also display a wide range of properties from spheroids with red $\mathrm{g}-\mathrm{i}$ colours $(\sim 0.8)$ to irregulars with blue blue $\mathrm{g}-\mathrm{i}$ colours $(\sim 0.3)$ (Trujillo et al. 2017). While faint, extended, low surface brightness galaxies are not a recent discovery, the Coma UDGs revealed for the first time their relative ubiquity in a dense environment. Moreover, compared to classical LSBs, the Coma UDGs were fainter and often more extended (Yagi et al. 2016; van der Burg et al. 2017).

Despite the relatively large number of reported UDGs, little is

\footnotetext{
* e-mail:sengupta.chandreyee@gmail.com(CS)

1 The effective radius of a galaxy is the radius at which half of the total light of the system is emitted.
}

known about their properties and formation scenario(s). Currently some of the principal ideas put forward to explain UDG formation are: (1) galaxies with higher than average angular momentum and low star formation (Amorisco \& Loeb 2016) (2) failed massive galaxies where either the environment or strong stellar feedback have supressed star formation (SF) (van Dokkum et al. 2016; Di Cintio et al. 2017) (3) a subset of classical LSBs or are a class of dwarf galaxies following an evolutionary sequence from field to cluster as suggested by Yozin \& Bekki (2015); Román \& Trujillo (2017); Carleton et al. (2019), i.e., normal dwarfs spirals which have been converted to UDGs by an environmentally aided process/processes (e.g., star formation (SF) quenching, tidal heating, tidal stripping or ram pressure).

In this paper we present resolved Giant Metrewave Radio Telescope (GMRT) H I observations of UGC 2162, a UDG with $\mu_{g}$ $\sim 24.4$ mag asec ${ }^{-2}, R_{e} \sim 1.7 \mathrm{kpc}$ and a $\mathrm{g}-\mathrm{i}$ colour of $0.33 \pm 0.02$ (Trujillo et al. 2017). Table 1 gives a summary of the galaxy's properties. Trujillo et al. (2017) showed that the stellar component of the galaxy consists of a elongated blue star forming region within a more extensive region of low surface brightness stellar emission. Using a redshift independent distance of $12.3 \pm 1.7 \mathrm{Mpc}$ for UGC 2162 it is claimed to be the nearest UDG (Trujillo et al. 2017), making it an excellent candidate for a resolved H I study. Being located in the IAC stripe82 Legacy Survey region, the galaxy has deep optical images. UGC 2162 is a member of the M 77 group and is projected $\sim 294 \mathrm{kpc}$ from the group's centre. The nearest group 
Table 1. Properties of UGC 2162

\begin{tabular}{|c|c|c|}
\hline Property ${ }^{a}$ & Units & Value \\
\hline $\mathrm{V}_{\text {radial(optical) }} b$ & {$\left[\mathrm{~km} \mathrm{~s}^{-1}\right]$} & $1185 \pm 6$ \\
\hline RA & [h:m:s] & 02:40:23.09 \\
\hline DEC & [d:m:s] & $+01: 13: 45.3$ \\
\hline Distance $^{c}$ & {$[\mathrm{Mpc}]$} & $12.3 \pm 1.7$ \\
\hline$R_{e}^{d}$ & {$[\mathrm{kpc}]$} & $1.7 \pm 0.2$ \\
\hline$\mu_{g}(0)^{e}$ & {$\left[\mathrm{mag} \operatorname{arcsec}^{2}\right]$} & $24.4 \pm 0.1$ \\
\hline $\mathrm{D}_{25}$ major axis & [arcsec] & 52 \\
\hline $\mathrm{D}_{25}$ major axis & {$[\mathrm{kpc}]$} & 3.2 \\
\hline Inclination $f$ & {$\left[{ }^{\circ}\right]$} & 55 \\
\hline Morphology & & UDG \\
\hline $12+\log (\mathrm{O} / \mathrm{H})^{g}$ & & $8.22 \pm 0.07$ \\
\hline$M_{*}^{h}$ & {$\left[10^{7} \mathrm{M}_{\odot}\right]$} & $2 \pm 2$ \\
\hline $\mathrm{M}_{H I}$ & {$\left[10^{7} \mathrm{M}_{\odot}\right]$} & 18 \\
\hline
\end{tabular}

$a$ From NED

$b$ From Hyperleda

$c$ See section 1 .

$d$ From Trujillo et al. (2017).

$e$ From Trujillo et al. (2017).

$f$ From BBarolo fit to medium resolution GMRT H i cube.

$g$ For the bightest SF knot from a SDSS spectrum Trujillo et al. (2017).

$h$ From Trujillo et al. (2017).

member to UGC 2162 with comparable or greater stellar mass is SDSS J023848.50+003114.2 which is projected 48.7 arcmin ( $175 \mathrm{kpc}$ ) away and separated in velocity by $277 \mathrm{~km} \mathrm{~s}^{-1}$.

Section 2 gives details of the GMRT observations, with observational results in section 3. A discussion follows in section 4 with a summary and concluding remarks in section 5 . In this paper we adopt the redshift to UGC 2162 of 0.00392 and distance to the galaxy of $12.3 \mathrm{Mpc}$ from Trujillo et al. (2017). We also adopt their angular scale of 1 arcmin $\sim 3.6 \mathrm{kpc}$. All $\alpha$ and $\delta$ positions referred to throughout this paper are $\mathrm{J} 2000.0$.

\section{OBSERVATIONS}

UGC 2162 was observed at $21 \mathrm{~cm}$ using the GMRT on the 8th March, 2018 and the observational parameters are detailed in Table 2. The GMRT data was analysed using the standard reduction procedures with the Astronomical Image Processing System (AIPS) software package. The flux densities are on the scale of Baars et al. (1977), with uncertainties of $\sim 5 \%$. After calibration and continuum subtraction in the uv domain the AIPS task IMAGR was used to convert the uv domain data to $\mathrm{H}_{\mathrm{i}}$ image cubes. Finally integrated $\mathrm{H}$, velocity field and velocity dispersion maps were extracted from the image cubes using the AIPS task MOMNT. To study the $\mathrm{H}$ I distribution in detail, image cubes with different resolutions were produced by applying different 'tapers' to the data with varying uv limits. Details of the final low, medium and high resolution maps are given in Table 2.

\section{OBSERVATIONAL RESULTS}

\subsection{H I morphology and $\mathrm{H}_{\mathrm{I}}$ mass}

Our UGC 2162 total intensity H I maps at low, medium and high angular resolution are shown in Figure 1, with the beam size and
Table 2. GMRT observational and map parameters

\begin{tabular}{ll}
\hline Rest frequency & $1420.4057 \mathrm{MHz}$ \\
Observation Date & 8 th March, 2018 \\
Integration time & $10.0 \mathrm{hrs}$ \\
primary beam & $24^{\prime}$ at $1420.4057 \mathrm{MHz}$ \\
Low resolution beam-FWHP & $43.3^{\prime \prime} \times 39.3^{\prime \prime}, \mathrm{PA}=13.4^{\circ}$ \\
Medium resolution beam-FWHP & $30.7^{\prime \prime} \times 28.8^{\prime \prime}, \mathrm{PA}=-4.5^{\circ}$ \\
High resolution beam-FWHP & $16.9^{\prime \prime} \times 12.9^{\prime \prime}, \mathrm{PA}=31.7^{\circ}$ \\
RA (pointing centre) & $02^{\mathrm{h}} 40^{\mathrm{m}} 23.10^{\mathrm{s}}$ \\
DEC (pointing centre) & $01^{\circ} 13^{\prime} 45.01^{\prime \prime}$ \\
\hline
\end{tabular}

PA for each resolution given in Table 2 and indicated with white ellipses on the maps. At the distance of $12.3 \mathrm{Mpc}$, the low, medium and high resolution full width half power (FWHP) beams sample the galaxy's H i disc at $2.6 \mathrm{kpc}, 1.8 \mathrm{kpc}$ and $1.0 \mathrm{kpc}$ respectively. While the two lower resolution maps show regular $\mathrm{H}$ i morphology, the high resolution map reveals sub-structure, with the two principal $\mathrm{H}_{\text {I }}$ maxima in that map having column densities of 1.0 to $1.2 \times 10^{21}$ atoms $\mathrm{cm}^{-2}$. Overall the outer $\mathrm{H}$ i disk morphology at all three resolutions is rather symmetric and shows no signs of extended tails or diffuse edge structures that would signify a recent interaction. From the low resolution H i map's major axis we estimate the $\mathrm{R}_{H I} \sim 86 \operatorname{arcsec}(\sim 5.2 \mathrm{kpc})$, compared to the $\mathrm{R}_{25}=26$ arcsec (1.6 kpc) from Trujillo et al. (2017). Its $\mathrm{R}_{H I} / \mathrm{R}_{25}$ is therefore $\sim 3.3$, i.e. almost twice the $\mathrm{R}_{H I} / \mathrm{R}_{25}$ of $\sim 1.8$ typical of late-type galaxies (Broeils \& Rhee 1997). Comparing the UGC $2162 \mathrm{R}_{H I} / \mathrm{R}_{25}$ to the morphologically unclassified Local Volume H I Survey (LVHIS Wang et al. 2017; Koribalski et al. 2018) sample (limited to galaxies with $\mathrm{M}_{*}<1 \times 10^{8} \mathrm{M}_{\odot}$ ), see Figure 3, indicates UGC 2162 lies within the upper range of $\mathrm{R}_{H I} / \mathrm{R}_{25}$ for observed galaxies with $\mathrm{M}_{*}<1 \times 10^{8} \mathrm{M}_{\odot}$. We note that the faint, patchy optical disk revealed by the deep g, r, i IAC Stripe 82 composite image, Figure 1 in Trujillo et al. (2017), extends to a radius of $\sim 60 \operatorname{arcsec}(3.6 \mathrm{kpc}$ ), i.e. more than twice the $\mathrm{R}_{25}$.

In Table 3 and Figure 2 we compare the properties of the integrated GMRT H i spectrum with those from the $42 \mathrm{~m}$ Green Bank Telescope (GBT, Springob et al. 2005) and Parkes Telescope Multibeam (HIPASS Meyer et al. 2004) single dish spectra. Both the $\mathrm{V}_{H I}$ and $\mathrm{W}_{20}$ derived from the three radio telescopes are in agreement within their uncertainties. While we believe our measurement of the $\mathrm{W}_{20}$ from HIPASS spectrum is correct, Meyer et al. (2004) reported the $\mathrm{W}_{20}$ from the HIPASS spectrum as $89 \mathrm{~km} \mathrm{~s}^{-1}$ and this is discussed in Section 4.1. The H I flux recovered by the GMRT interferometric observation $\left(\mathrm{S}_{H I}=4.5 \mathrm{Jy} \mathrm{km} / \mathrm{s}\right)$ is in good agreement the GBT absorption corrected flux $\left(\mathrm{S}_{H I}=5.1 \mathrm{Jy} \mathrm{km} / \mathrm{s}\right)$. Because it has the best signal to noise ratio $(\mathrm{S} / \mathrm{N})$ of the available spectra, we use the GBT flux to estimate the galaxy's Hi mass. This gives a $\mathrm{M}_{H I}=1.8 \times 10^{8} \mathrm{M}_{\odot}$ for the galaxy.

From the IAC Stripe82 deep observations (Trujillo et al. 2017) estimated the UGC $2162 \mathrm{~g}$ - band optical diameter $\mathrm{D}_{25} \sim 52 \operatorname{arcsec}$ $(3.2 \mathrm{kpc})$. Using this optical diameter, the $\log \left(\mathrm{M}_{H I} / D_{l}{ }^{2}\right)$ value for UGC 2162 is 7.24. The average value of $\log \left(\mathrm{M}_{H I} / D_{l}{ }^{2}\right)$ for galaxies of similar morphological type is $6.87 \pm 0.17$ (Haynes \& Giovanelli 1984), indicating UGC 2162 is H I rich compared to other galaxies of similar size and morphological type. This conclusion is confirmed in Figure 4 which shows $\mathbf{M}_{\text {gas }} / \mathbf{M}_{*}$ compared to $\mathbf{M}_{*}$ for UGC 2162, the LITTLE THINGS sample of dwarf galaxies ${ }^{2}$ (Oh et al. 2015) and the morphologically unclassified LVHIS sam-

2 for which $\mathrm{M}_{*}$ values available 


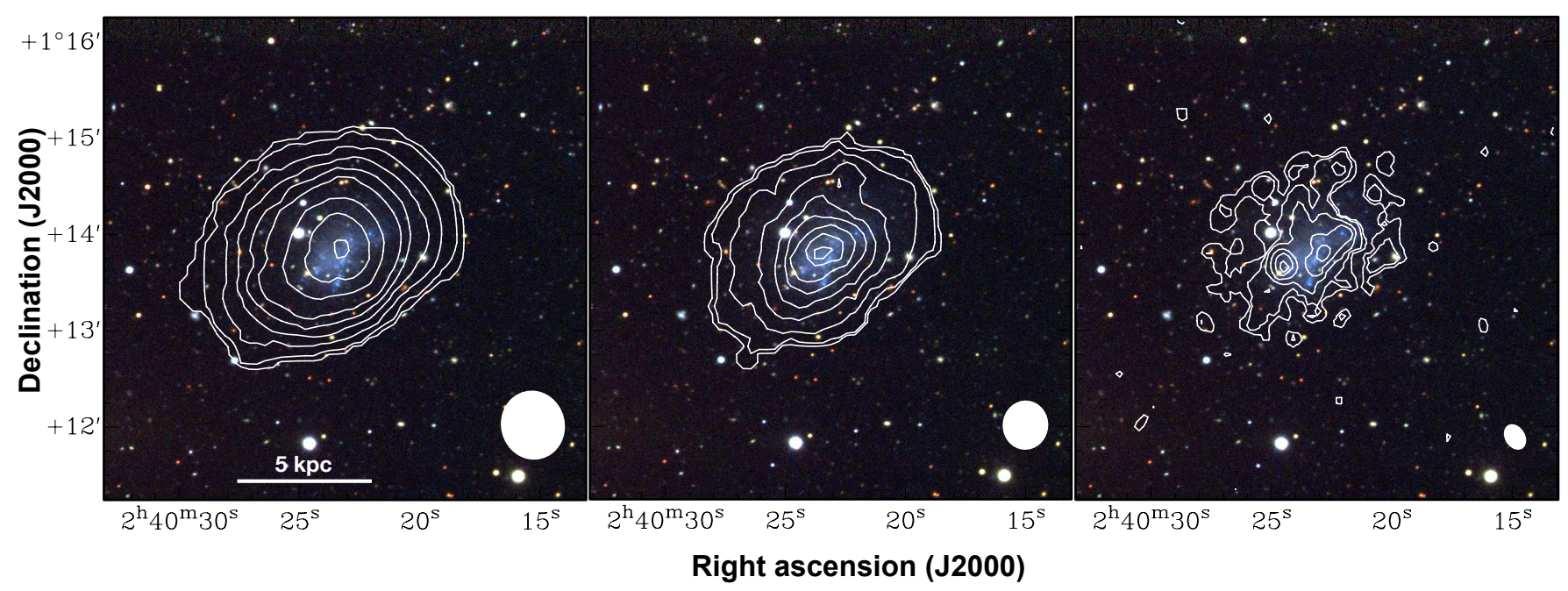

Figure 1. UGC 2162: left to right GMRT low $\left(43.3^{\prime \prime} \times 39.3^{\prime \prime}\right)$, medium $\left(30.7^{\prime \prime} \times 28.8^{\prime \prime}\right)$ and high $\left(16.9^{\prime \prime} \times 12.9^{\prime \prime}\right)$ resolution integrated H map contours on SDSS g, r, i band composite image. The GMRT beam size and orientation is shown with white ellipses at the bottom right of each panel. Low resolution contour levels: $10^{20}$ atoms cm$~_{-2}(0.3,0.7,1.3,1.9,2.6,3.2,4.5,5.6,7.1)$ Mid resolution contour levels: $10^{20}$ atoms cm $\mathrm{cm}^{-2}(0.5,0.9,1.8,3.1,4.3,5.6,6.8$, 8.1, 8.9) High resolution contour levels: $10^{20}$ atoms $\mathrm{cm}^{-2}(1.2,2.5,5.0,7.5,10.0,12.6)$.

Table 3. UGC 2162 GMRT, GBT and HIPASS H I spectra properties

\begin{tabular}{llrrrr}
\hline Telescope & $\begin{array}{l}\text { Velocity } \\
\mathrm{km} \mathrm{s}^{-1}\end{array}$ & $\begin{array}{r}\mathrm{W}_{20} b \\
\mathrm{~km} \mathrm{~s}^{-1}\end{array}$ & $\begin{array}{r}\text { Channel width } \\
\mathrm{km} \mathrm{s}^{-1}\end{array}$ & $(\mathrm{~S}) / \mathrm{N}$ & $\begin{array}{r}\mathrm{S}_{H I} \\
\mathrm{Jy} \mathrm{km} / \mathrm{s}\end{array}$ \\
\hline GMRT $^{c}$ & $1185 \pm 2$ & $49 \pm 5$ & 6.9 & 5.6 & 4.5 \\
GBT & $1182 \pm 1$ & $50 \pm 2$ & 2.1 & 8.7 & $5.1^{d}$ \\
HIPASS & $1178 \pm 2$ & $53 \pm 4$ & 13.4 & 6.4 & $5.4^{e}$ \\
Optical & $1185 \pm 6$ & & & & \\
\hline
\end{tabular}

\footnotetext{
$a$ Velocity $=\mathrm{V}_{H I}$ except for 'Optical' which is the optical velocity from NED.

$b$ Our measurement from the GBT and HIPASS spectra.

$c$ From the GMRT mid-resolution cube.

$d$ Self-absorption corrected, integrated flux density from (Springob et al. 2005).

$e$ From (Meyer et al. 2004).
}

ple for galaxies, but limited to members of both samples with $\mathbf{M}_{*}$ $<1 \times 10^{8} \mathrm{M}_{\odot}$. For consistency with LITTLE THINGS, $\mathrm{M}_{\text {gas }}$ for UGC 2162 and LVHIS $=1.4 \times \mathrm{M}_{\mathrm{HI}}$, with the additional factor to account for molecular gas (0.04) and $\mathrm{He}(0.36)$. The figure shows that the $\mathrm{M}_{\text {gas }} / \mathrm{M}_{*}$ ratio for UGC 2162 is 13.3 , which is almost a factor of three higher than the median $\mathrm{M}_{\text {gas }} / \mathrm{M}_{*}$ ratio for the LITTLE THINGS dwarfs of 4.7 and the mean of $4.6 \pm 5.0$ for LVHIS. The LITTLE THINGS outlier with $\mathbf{M}_{\text {gas }} / \mathbf{M}_{*}>40$ is the well known super gas rich dwarf DDO154. Figure 4 also shows the mean $\mathrm{M}_{\text {gas }} / \mathrm{M}_{*}$ ratio $^{3}$ (7.18) from the 21 UDGs in the NIHAO simulation (Di Cintio et al. 2017). From the figure we see that the UGC 2162 $\mathrm{M}_{\text {gas }} / \mathrm{M}_{*}$ ratio is higher than the mean of the simulated UDGs. (see also Figure 3). Overall our analysis suggests that UGC 2162 falls within the ranges of $\mathrm{R}_{H I} / \mathrm{R}_{25}$ and $\mathrm{M}_{H I} / \mathrm{M}_{*}$ observed in galaxies

${ }^{3} \mathrm{M}_{\text {gas }}=\mathrm{M}_{H I} \times 1.4$ with similar $\mathbf{M}_{*}$ in the THINGS and LVHIS samples, but displays amongst the highest $\mathrm{M}_{\text {gas }} / \mathrm{M}_{*}$ ratios in its $\mathrm{M}_{*}$ range.

Figure 5 compares the $\mathrm{MH} \mathrm{i} / \mathrm{M}_{*} \mathrm{v} \mathrm{R}_{e}$ and $\mathrm{g}-\mathrm{i}$ color $\mathrm{v} \mathrm{R}_{e}$ for UGC 2162 with those from samples of UDGs with $\mathrm{H}_{\mathrm{I}}$ single dish detections in ALFALFA (Leisman et al. 2017) and H I mapping (Spekkens \& Karunakaran 2018). MH I/M $\mathbf{M}_{*}$ for UGC 2162 and the Spekkens sample was calculated using $\mathrm{M}_{*}$ from (Trujillo et al. 2017) and Spekkens \& Karunakaran (2018) respectively. $\mathbf{M}_{*}$ was calculated for the Leisman ${ }^{4}$ sample using SDSS model magntudes ( $\mathrm{g}$ and $\mathrm{i}$ band) and parameters from Bell et al. (2003) and Blanton et al. (2003). Additionally, we calculated the SDSS g -

4 The full sample contained 115 UDGs but the we excluded 19 UDGs with $\mathrm{MH} \mathrm{I} / \mathrm{M}_{*} \$>80$. The 19 excluded UDGs are outliers in terms of $\mathrm{MH} \mathrm{s} / \mathrm{M}_{*}$ with $\mathrm{g}$ band magnitudes in most cases $\geqslant 21$. These galaxies were excluded because the extreme $\mathrm{MH} \mathrm{I} / \mathrm{M}_{*}$ raised questions about the reliability of their SDSS photometry for determining $\mathbf{M}_{*}$. 


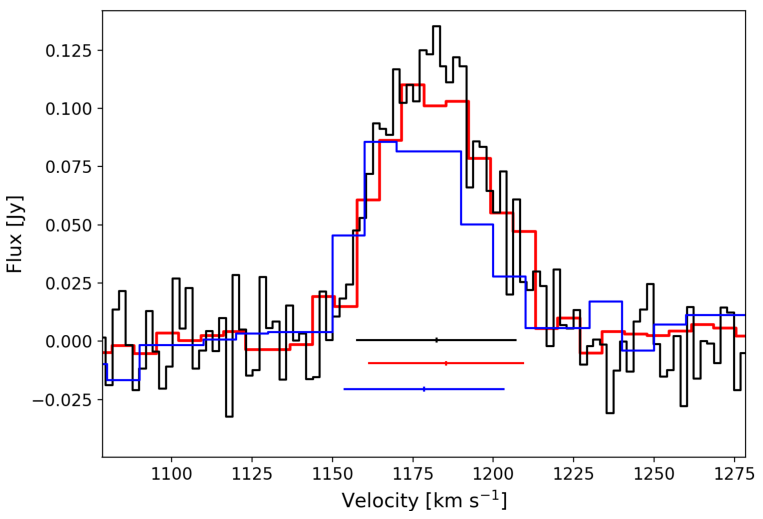

Figure 2. UGC 2162 H I spectra: GBT (black), GMRT (red) and HIPASS (blue). The channel widths of the GBT, GMRT and HIPASS spectra are 2.1, 6.9 and $13.4 \mathrm{~km} \mathrm{~s}^{-1}$ respectively The bars at the base of the spectra show the $\mathrm{W}_{20}$ of each spectrum in its respective colour.

i colours for all galaxies from their SDSS model magnitudes, although the UGC 2162 magnitudes were from Trujillo et al. (2017). Magnitudes used for calculating the $\mathrm{g}-\mathrm{i}$ colours and $\mathrm{M}_{*}$ for the Leisman sample were corrected for galactic extinction using the Schlafly \& Finkbeiner (2011) values from NED extinction calculator. From Figure 5 we see that UGC 2162 has amongst the smallest $\mathrm{R}_{e}$ of the UDGs with $\mathrm{H}_{\mathrm{I}}$ detections. However, its $\mathrm{MH} \mathrm{s} / \mathrm{M}_{*}$ of 9.0 while not as extreme as some Leisman UDGs is above that samples typical value. On the other hand UGC 2162's g - i colour ${ }^{5}(0.243)$ is slightly bluer than most of the Leisman sample, which probably reflects the current enhancement in SFR noted by Trujillo et al. (2017). The H I rich nature of UGC 2162 is also confirmed in comparisison with the simulated UDGs from Di Cintio et al. (2017), see Figure 4. This figure shows UGC 2162 to have a smaller $\mathrm{R}_{e}$ and be gas rich compared to an average simulated Di Cintio et al. (2017) UDG.

\subsection{H I kinematics}

Figure 6 summarises the results from our analysis of UGC $2162 \mathrm{H} \mathrm{I}$ kinematics. The upper panel of Figure 6 shows the $\mathrm{H}_{\text {I velocity field }}$ from medium resolution cube $(30.7$ " $\times 28.9$ " beam $)$, which has the characteristics of a rotating disc with a moderate rotation velocity and a signature of a warp in the SE. The black contours at the centre of this panel are the two highest $\mathrm{H}_{\text {I }}$ column density contours from the medium resolution total intensity $\mathrm{H}_{\text {I }}$ map. These $\mathrm{H}_{\text {I }}$ contours coincide with the kinematic centre as well as the optical centre of the galaxy. The velocity field of UGC 2162 show no significant sign of recent major interaction. The position velocity (PV) diagram for a slice taken along the $\mathrm{H}_{\mathrm{I}}$ major axis, position angle $(\mathrm{PA})=307^{\circ}$, is shown in the middle panel of Figure 6 and confirms a rotating disc. A small mass of low density extra-planar $\mathrm{H}_{\mathrm{I}}$ is seen $\sim 15-20$ arcsec $(\sim 1 \mathrm{kpc}) \mathrm{SE}$ of the kinematic centre in the PV diagram. The lower panel of Figure 6 shows the rotation curve extracted from BBAROLO (Di Teodoro \& Fraternali 2015) fit to the medium resolution cube. The figure shows a slowly rising rotation curve which is a common feature for dwarf galaxies. The BBAROLO best fit gives an inclination

\footnotetext{
${ }^{5}$ Corrected for galaxtic extinction.
}

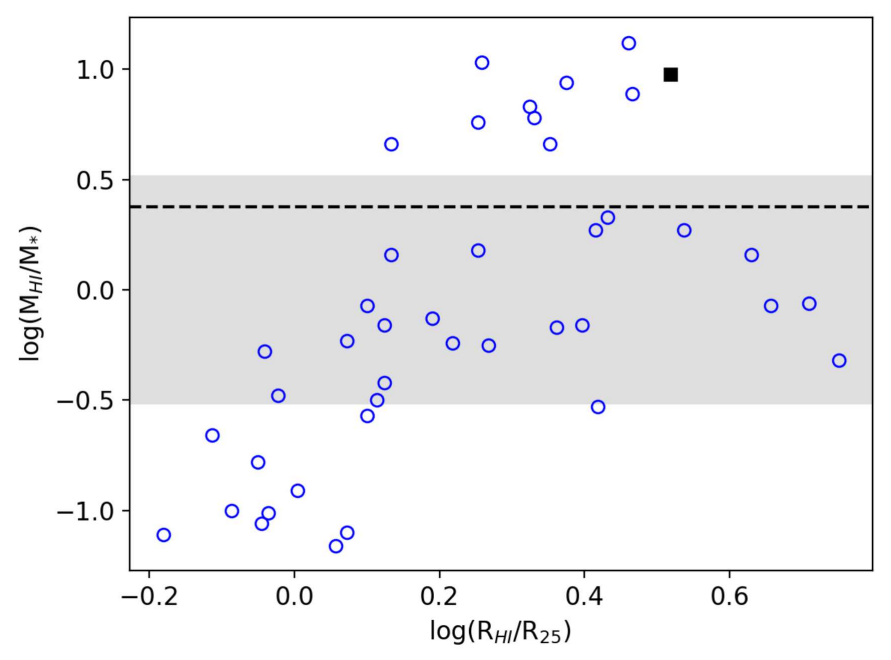

Figure 3. $\log \left(\mathbf{M}_{H I} / \mathbf{M}_{*}\right) \mathbf{v} \log \left(\mathbf{R}_{H I} / \mathbf{R}_{25}\right)$ : UGC 2162 (black square) and LVHIS local galaxies (limited to galaxies with $\mathrm{M}_{*}<1 \times 10^{8} \mathrm{M}_{\odot}$ ) from Wang et al. (2017) and Koribalski et al. (2018) - (blue circles). The dashed line is the mean $\log \left(\mathrm{M}_{H I} / \mathrm{M}_{*}\right)$ for 21 UDGs in the NIHAO simulation (with the shaded area showing its uncertainty) from Di Cintio et al. (2017).

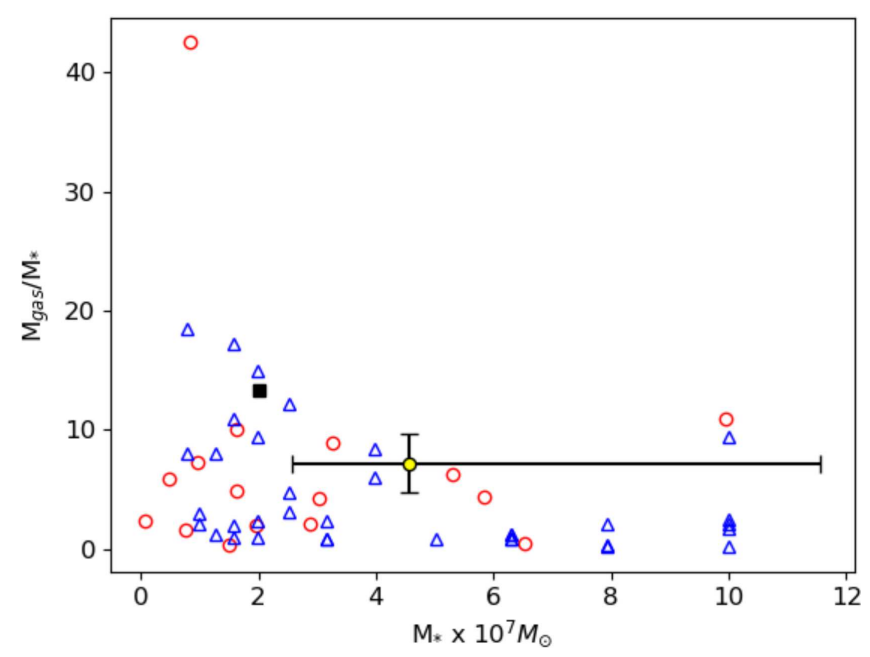

Figure 4. $\mathbf{M}_{\text {gas }} / \mathbf{M}_{*} \mathbf{v} \mathbf{M}_{*}$ : UGC 2162 (black square), LITTLE THINGS dwarf galaxies (red circles) from Oh et al. (2015) and LVHIS local galaxies from Wang et al. (2017) and Koribalski et al. (2018) - (blue triangles). The yellow filled circle is the mean $\mathrm{M}_{\text {gas }} / \mathrm{M}_{*} \vee \mathrm{M}_{*}$ for 21 UDGs in the NIHAO simulation from Di Cintio et al. (2017).

of 55.5 , with $\mathrm{V}_{\text {rot }}$ at the outermost fitted ring, $\mathrm{r}=67.5 \operatorname{arcsec}(4.05$ $\mathrm{kpc}) \sim 25 \mathrm{~km} \mathrm{~s}^{-1}$. The $\mathrm{M}_{d y n}$ enclosed within each ring fitted with BBAROLO is used in section 4.1 to estimate the galaxy's virial mass $\left(\mathrm{M}_{v i r}\right)$. We also estimated the $\mathrm{V}_{\text {rot }}$ using the $\mathrm{H}_{\mathrm{I}} \mathrm{W}_{20}$ (adjusted for the inclination from the BBAROLo model fit) where $\mathrm{V}_{\text {rot }}=0.5 \Delta \mathrm{V}$ $\left[\mathrm{km} \mathrm{s}^{-1}\right] / \sin (\mathrm{i})$ with $\Delta \mathrm{V}=\mathrm{W}_{20}\left(50 \mathrm{~km} \mathrm{~s}^{-1}\right)$ from the GBT spectrum 

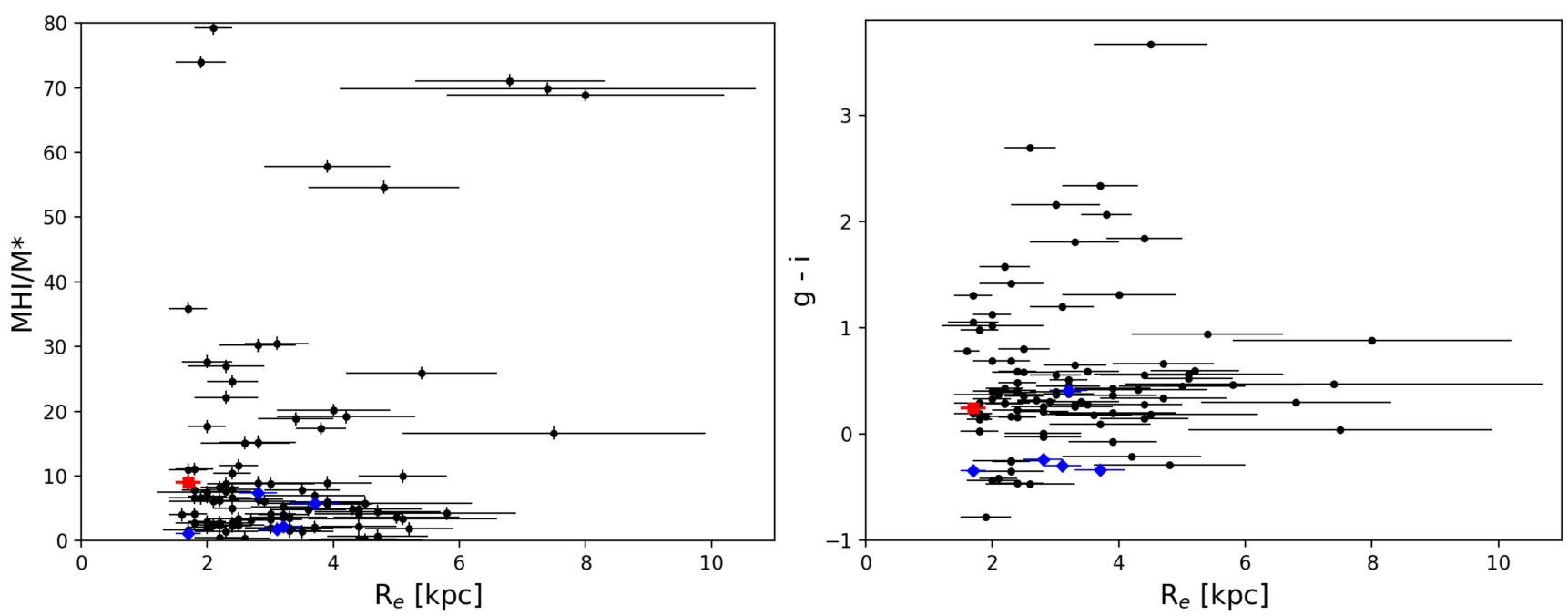

Figure 5. MHI/M $\mathbf{M}_{*} \mathbf{v} \mathbf{R}_{e}$ UGC 2162 [red square] in comparison with UDG samples with $\mathrm{H}$ I detections; H I-bearing ultra-diffuse ALFALFA sources (HUDS) from Leisman et al. (2017) [black dots] and H I mapping from Spekkens and Karunakaran (2018) [blue diamonds].

and $\mathrm{i}=55.5^{\circ} \pi / 180$. This second method gives $\mathrm{V}_{\text {rot }} \sim 30.8 \mathrm{~km} \mathrm{~s}^{-1}$ at the outer edge of the detected $\mathrm{H}_{\mathrm{I}}$ disk. These values are also used in the section 4.1 analysis.

In Figure 7 we compare the $\mathrm{V}_{\text {rot }}$ from for UGC 2162 with the $\mathrm{V}_{\text {rot }}$ derived from model fits to the VLA $\mathrm{H}_{\text {I }}$ for the LITTLE THINGS dwarf galaxies by Oh et al. (2015). The plot shows that the UGC $2162 \mathrm{~V}_{\text {rot }}=30.8 \mathrm{~km} \mathrm{~s}^{-1}$, derived from the GBT W 20 , falls within the range of $\mathrm{V}_{\text {rot }}$ for LITTLE THINGS with similar $\mathrm{M}_{\mathrm{HI}}$ and below the LITTLE THINGS median $\mathrm{V}_{\text {rot }}$ of $39.5 \mathrm{~km} \mathrm{~s}^{-1}$.

A galaxy's $A_{f u x}$ ratio is a measure of the asymmetry in its integrated $\mathrm{H}_{\mathrm{I}}$ flux density profile (within its $\mathrm{W}_{20}$ velocity range) at velocities above and below the galaxy's systemic velocity, $V_{\mathrm{HI}}$. Even isolated late-type galaxies display a scatter of $A_{f l u x}$ ratio, which is well characterised by a half Gaussian, with its mean equal to 1.0 and a $1 \sigma$ dispersion of 0.13 . This half Gaussian was obtained from a fit to the distribution of $A_{f l u x}$ values from a sample of AMIGA ${ }^{6}$ isolated galaxies (Espada et al. 2011). The value of an $A_{f u x}$ deviating by $1 \sigma$ from the mean of that distribution is then 1.13. A study by Scott et al. (2018) indicated that $A_{f l u x}$ is quite sensitive to recent $(\lesssim 0.7 \mathrm{Gyr})$ interactions which impact H I disks. For UGC 2162 $A_{\text {flux }}=1.07 \pm 0.13$, measured from the GBT H I spectrum, which is within the $1 \sigma$ value from the isolated sample. UGC 2162's $\mathrm{H}_{\mathrm{I}}$ profile is therefore consistent with a symmetric morphology. Both the $\mathrm{H}_{\mathrm{I}} A_{f l u x}$ and the velocity field indicate a relatively unperturbed rotating $\mathrm{H}_{\mathrm{I}}$ disk which makes it suitable for use in determining the galaxy's $\mathrm{M}_{d y n}$ and $\mathrm{M}_{v i r}$. Overall UGC 2162's H I kinematics indicate a moderately rotating disk viewed at an inclination of $55.5^{\circ}$. A comparison with the LITTLE THINGS sample of dwarf galaxies indicates its $\mathrm{V}_{\text {rot }}$ of $30.8 \mathrm{~km} \mathrm{~s}^{-1}$ falls within the range of $\mathrm{V}_{\text {rot }}$ for LITTLE THINGS galaxies with similar $\mathrm{M}_{\mathrm{HI}}$, but below the median $\mathrm{V}_{\text {rot }}$ for LITTLE THINGS galaxies.

${ }^{6}$ Analysis of the interstellar Medium of Isolated GAlaxies

\section{DISCUSSION}

The main goals of our Hi observations of UDGs are to improve knowledge of the nature of their dark matter halos and to constrain UDG formation scenarios. In this section we consider our results for UGC 2162.

\subsection{Dark matter halo of UGC 2162}

As a key driver of UDG formation and evolution, their DM mass and its distribution has been debated and investigated using models and globular cluster velocity dispersions observations (e.g., van Dokkum et al. 2016; Toloba et al. 2018) as well as single dish H i spectra (e.g. Trujillo et al. 2017; Spekkens \& Karunakaran 2018). Seeing faint UDGs surviving in clusters, van Dokkum et al. (2015a,b) predicted them to be dark matter dominated galaxies. A subsequent velocity dispersion measurement and globular cluster count for DF44 (a Coma cluster UDG, with luminosity $\mathrm{L}_{v} \sim$ $2 \times 10^{8} \mathrm{~L}_{\odot}$ ) indicated a MW size DM halo with $\mathrm{M}_{h} \sim 10^{12} \mathrm{M}_{\odot}$ (van Dokkum et al. 2016). More recent studies show that, while high dynamical to stellar mass ratios are common in UDGs, e.g. VCC1287, DF17 and 18 Coma UDGs, typically dwarf scale halos are inferred (Beasley \& Trujillo 2016; Amorisco \& Loeb 2016). Zaritsky (2017), using scaling relations, suggested that UDGs may span a range of halo masses between large spirals and dwarf galaxies, though no observations have been carried out to verify these predictions. Additionally, several observational studies of UDGs have used globular cluster velocity dispersions to estimate their DM content (e.g. Beasley et al. 2016; van Dokkum et al. 2018; Toloba et al. 2018). Biases related to the use of globular clusters to determine UDG DM halo masses are discussed in Laporte et al. (2019). However, resolved H I can probe DM halo properties to much larger radii than globular clusters. Therefore, at least for gas rich UDGs $\mathrm{H}_{\mathrm{I}}$ is better suited to investigating the critical questions of a UDG's DM mass and its distribution.

The $\mathrm{H}$ I rotation curves of massive spiral galaxies are observed to initially rise steeply but quickly become flat and remain flat in 

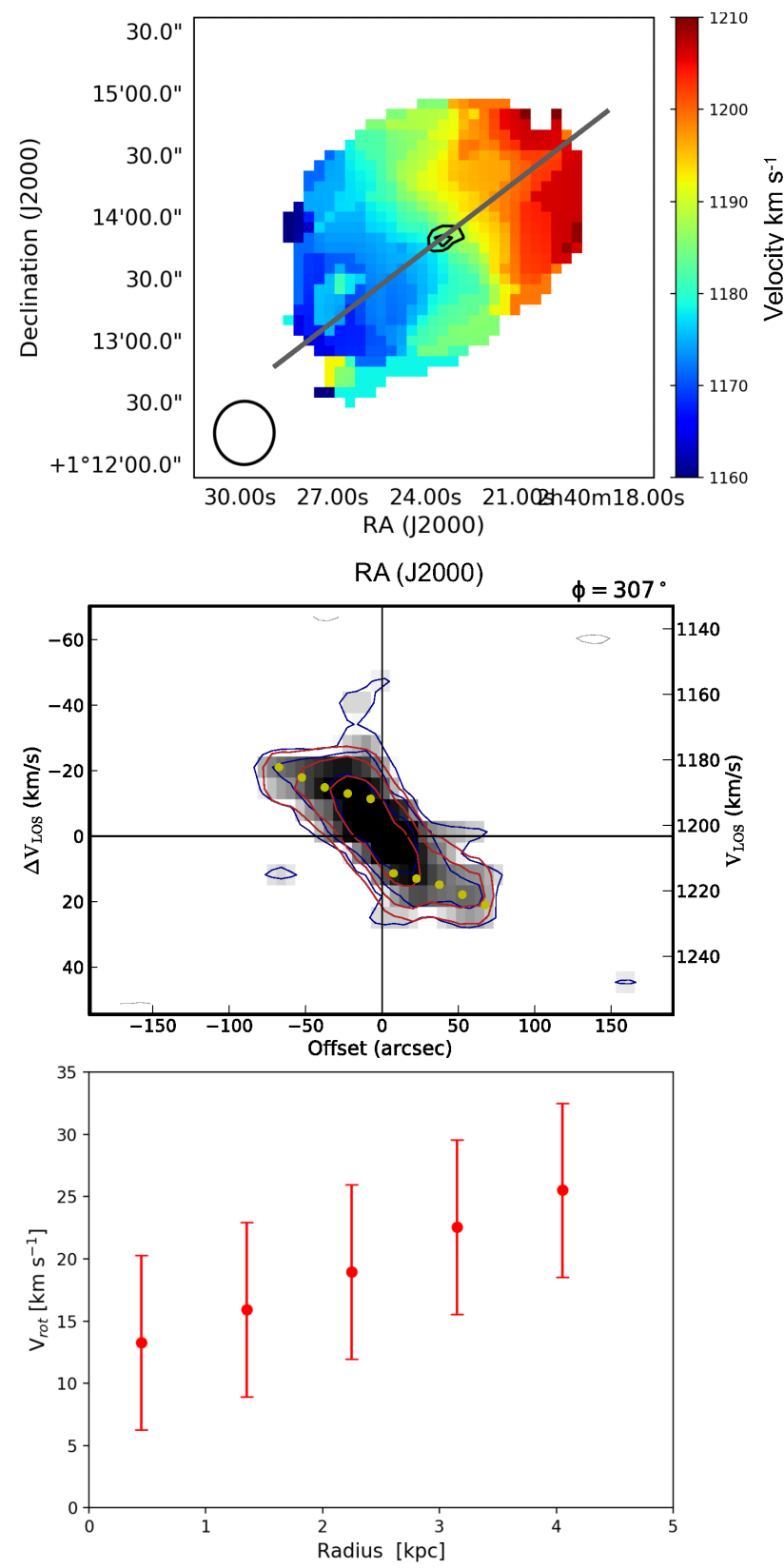

Figure 6. UGC 2162 H I kinematics: Top: GMRT velocity field from the medium resolution cube. The two highest column density contours from the medium resolution $\mathrm{H}_{\mathrm{I}}$ integrated map are shown in black. The PV diagram (panel below) slice $\mathrm{PA}=307^{\circ}$ is shown with a grey line. Middle: $\mathrm{PV}$ diagram from a PA $=307^{\circ}$ slice. Positive positional offsets are to the NW. The blue contours are from the data and the red are from the BBAROLo best fit model. Bottom: Rotation curve derived from the BBAROLo five ring model fit.

the outer regions. In contrast dwarf galaxies' $\mathrm{H}_{\mathrm{I}}$ rotation curves are observed to rise more slowly without necessarily flattening out (Di Teodoro \& Fraternali 2015) and this is the trend observed in the UGC $2162 \mathrm{H}$ I rotation curve, see Figure 6. We used the $\mathrm{V}_{\text {rot }}$ and radius for each of the 5 model rings fitted by BвARoLo to the

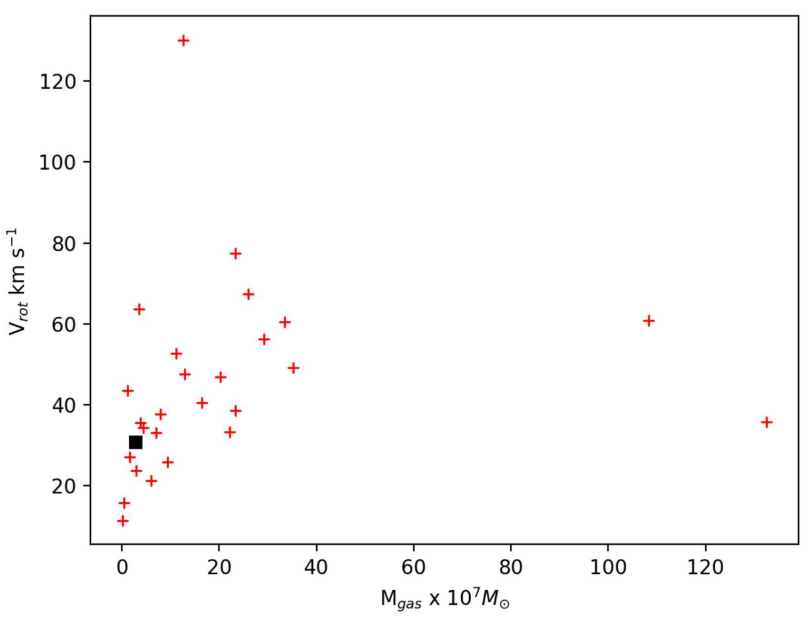

Figure 7. $\mathbf{V}_{\text {rot }} \vee M_{\text {gas }}$ : UGC 2162 (Black square) and LITTLE THINGS dwarf galaxies (red crosses) from Oh et al. (2015). The UGC 2162, $\mathrm{V}_{\text {rot }}$ $=30.8 \mathrm{~km} \mathrm{~s}^{-1}$ is derived from the GBT $\mathrm{W}_{20}$ in section 3.2. For both UGC 2162 and the LITTLE THINGs galaxies $\mathrm{M}_{\text {gas }}=1.4 \times \mathrm{M}_{\mathrm{HI}}$ with the additional factor to account for molecular gas and $\mathrm{He}$.

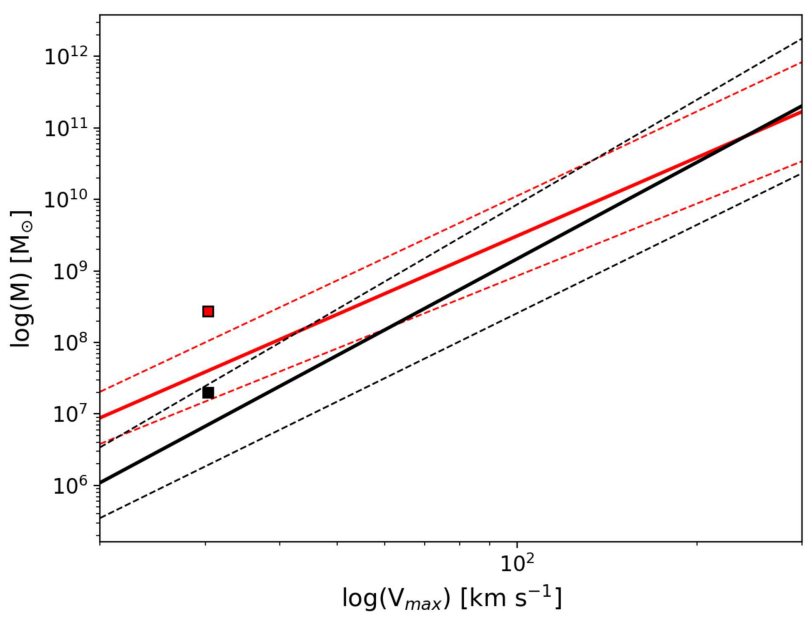

Figure 8. UGC 2162 Tully-Fisher relations: The position of UGC 2162 is shown with a red square relative to the Baryonic Tully-Fisher Relation (red solid line). Also shown is UGC 2162's position (black square) relative to the Stellar Tully-Fisher Relation (black solid line). The dashed lines in each colour indicate the $1 \sigma$ scatted in their respective Tully-Fisher Relation.

medium resolution GMRT H I cube together with equation 1 below to calculate the $\mathrm{M}_{d y n}$ enclosed within the radius of each ring:

$$
M_{d y n}=V_{r o t}^{2} r_{H I} / G\left[M_{\odot}\right]
$$

For UGC 2162 the BBAROLo model ring with the largest radius from the $\mathrm{H}_{\mathrm{I}}$ kinematic centre was at $\mathrm{r}_{H I}=67.5 \operatorname{arcsec}(\sim 4.05 \mathrm{kpc})$. Using this rings's $\mathrm{H}_{\mathrm{I}}$ extent and $\mathrm{V}_{\text {rot }}=25.5 \mathrm{~km} \mathrm{~s}^{-1}$, we estimate the dynamical mass $\left(\mathrm{M}_{d y n}\right)$ within the inner $4.05 \mathrm{kpc}$ using equation 1 as $\sim 0.6 \times 10^{9} \mathrm{M}_{\odot}$. Additionally, we calculated $\mathrm{M}_{d y n}=1.14$ 
${ }_{-0.47}^{+0.57} \times 10^{9} \mathrm{M}_{\odot}$ enclosed within the $\mathrm{R}_{H I} \sim 86 \operatorname{arcsec}(5.2 \mathrm{kpc})$, estimated from the low resolution GMRT map and $\mathrm{V}_{\text {rot }}=30.8 \mathrm{~km} \mathrm{~s}^{-1}$ derived in Section 3.2 from the GBT $\mathrm{W}_{20}$. Our $\mathrm{M}_{d y n}$ value (1.14 $\times 10^{9} \mathrm{M}_{\odot}$ ) enclosed within the $5.2 \mathrm{kpc} \mathrm{R}_{H I}$ is only about $25 \%$ of the $\mathrm{M}_{d y n}=4.6 \times 10^{9} \mathrm{M}_{\odot}$ based on a guessed at, but similar, $\mathrm{R}_{H I}$ reported in Trujillo et al. (2017). The difference is principally due to their adoption of an inclination corrected $\mathrm{V}_{\text {rot }}=64 \mathrm{~km} \mathrm{~s}^{-1}$ based on a HIPASS $\mathrm{W}_{20}=89 \mathrm{~km} \mathrm{~s}^{-1}$ from Meyer et al. (2004). The Meyer value is nearly twice the GBT W $\mathrm{W}_{20}$ of $50 \pm 2 \mathrm{~km} \mathrm{~s}^{-1}$ we have adopted. But Table 2 shows our re-measurement of the HIPASS $\mathrm{W}_{20}$ is in agreement, within the uncertainties, with both the GBT and GMRT $\mathrm{W}_{20}$ values.

Figure 9 shows, with red open circles, the $\mathbf{M}_{d y n}$ enclosed within the five BвARoLo model radii of 7.5", 22.5", 37.5", 52.5" and 67.5" $(0.45,1.35,2.25,3.15$ and $4.05 \mathrm{kpc})$ from the $\mathrm{H}_{\mathrm{I}}$ kinematic centre using equation 1 . The $\mathrm{M}_{d y n}=1.14_{-0.47}^{+0.57} \times 10^{9} \mathrm{M}_{\odot}$ enclosed within the $\mathrm{R}_{H I} \sim(5.2 \mathrm{kpc})$ is shown in the figure with a black square. The figure also shows Navarro-Frenk-White (NFW) model $^{7}$ cumulative masses for DM halos with $\mathrm{M}_{200}=5 \times 10^{10} \mathrm{M}_{\odot}$ (red dashed curve) and $\mathrm{M}_{200}=8.8 \times 10^{10} \mathrm{M}_{\odot}$ (black solid curve). The respective $\mathrm{V}_{\max }$ from these halo models are $51.1 \mathrm{~km} \mathrm{~s}^{-1}$ and $64.2 \mathrm{~km} \mathrm{~s}^{-1}$ with the respective $\mathrm{R}_{200} \mathrm{~S}$ of $228 \mathrm{kpc}$ and $275 \mathrm{kpc}$. A concentration parameter $=2$ was used for both NFW models. The two NFW models fit the $\mathrm{M}_{d y n}$ enclosed within ring radii derived from BBAROLO and the low resolution $\mathrm{H}_{\mathrm{I}}$ map disk edge radius, provide a likely range for the UGC $2162 \mathrm{DM}$ halo properties. Figure 10 shows the infered $\mathrm{M}_{200}$ for UGG 2162 (mean from two NFW halo models) $=6.9 \times 10^{10} \mathrm{M}_{\odot}$, which is a factor of three higher than the median $\mathrm{M}_{200}\left(2.1 \times 10^{10} \mathrm{M}_{\odot}\right)$ for the LITTLE THINGS dwarf galaxies from model fits carried out by Oh et al. (2015). Additionally, UGC 2162's rotation curve is consistent with the slowly rising rotation curves observed in dwarf and LSB galaxies (e.g. Oh et al. 2015).

To summarise, we estimate the UGC 2162's $\mathrm{M}_{d y n}$ is $\sim 1.14$ $\times 10^{9} \mathrm{M}_{\odot}$ within the $\mathrm{R}_{H I}(5.2 \mathrm{kpc})$, derived from the low resolution Hi map. Halo model fits to both this and $\mathbf{M}_{d y n}$ derived from BBAROLO ring model fits infer $\mathrm{M}_{200}$ in the range 5.0-8.8 $\times 10^{10} \mathrm{M}_{\odot}$, which is slightly higher than the median for LITTLE THINGS galaxies. UGC 2162 also displays a slowly rising H I rotation curve, typical of dwarf galaxies. UDGs encompass a range of different types of galaxies from dark matter dominated (eg. DF44: van Dokkum et al. (2016) to lacking in dark matter (eg. 1052-DF2: Danieli et al. (2019)). In that spectrum of galaxies, a comparison of the physical properties, $\mathrm{M}_{200}$, $\mathrm{H}_{\mathrm{I}}$ velocity curve profiles and $\mathrm{V}_{\text {rot }}$ reported for LITTLE THINGS dwarf galaxies, lead us to conclude that the baryonic component of UGC 2162 is inhabiting a dark matter halo with a mass and profile characteristic of a dwarf galaxy.

\subsection{Formation scenario}

As noted in Section 1, there are multiple hypotheses in the literature regarding formation of UDGs. It is also not clear if an umbrella term UDG should be used for these galaxies which may be in reality a collection of galaxies of all sizes, morphologies and stages of evolution with the only common factor being their extremely faint central surface brightness. We discuss here possible formation scenarios of the dwarf UDG, UGC 2162.

As shown in Figure 4 and described in Section 3.1 the $\mathrm{M}_{\text {gas }} / \mathrm{M}_{*}$

7 Halotools (Hearin et al. 2017)

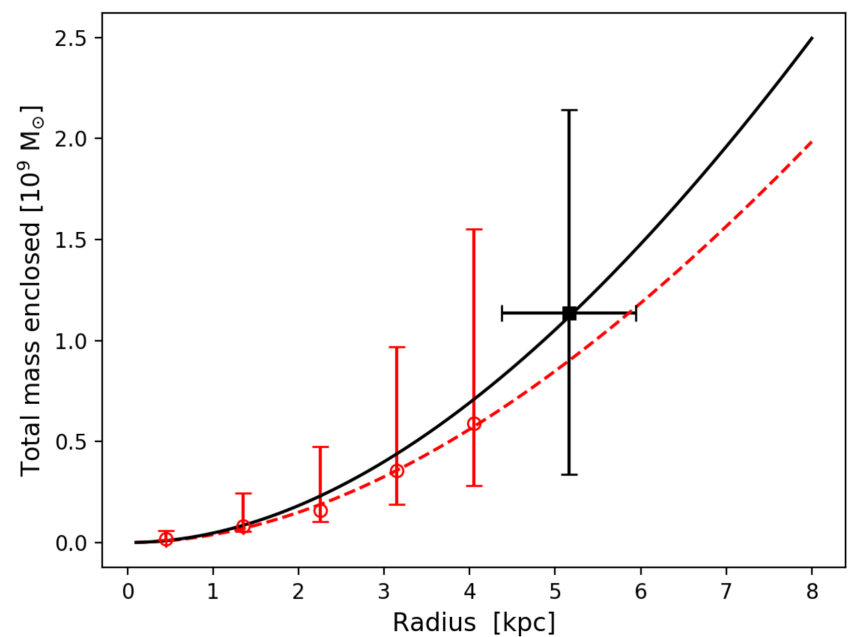

Figure 9. UGC 2162: $\mathrm{M}_{d y n}$ interior to the five ring BBAROLO H I model fits (red open circles) and $\mathrm{M}_{d y n}$ derived from the $\mathrm{R}_{H I}$ from low resolution GMRT $\mathrm{H}_{\mathrm{I}}$ map and $\mathrm{W}_{20}$ (black square). Also shown are NFW model cumulative masses for DM halos with $\mathrm{M}_{200}=5 \times 10^{10} \mathrm{M}_{\odot}$ (red dashed curve) and $\mathrm{M}_{200}=8.8 \times 10^{10} \mathrm{M}_{\odot}$ (black solid curve). The concentration parameter $=2$ for both NFW models.

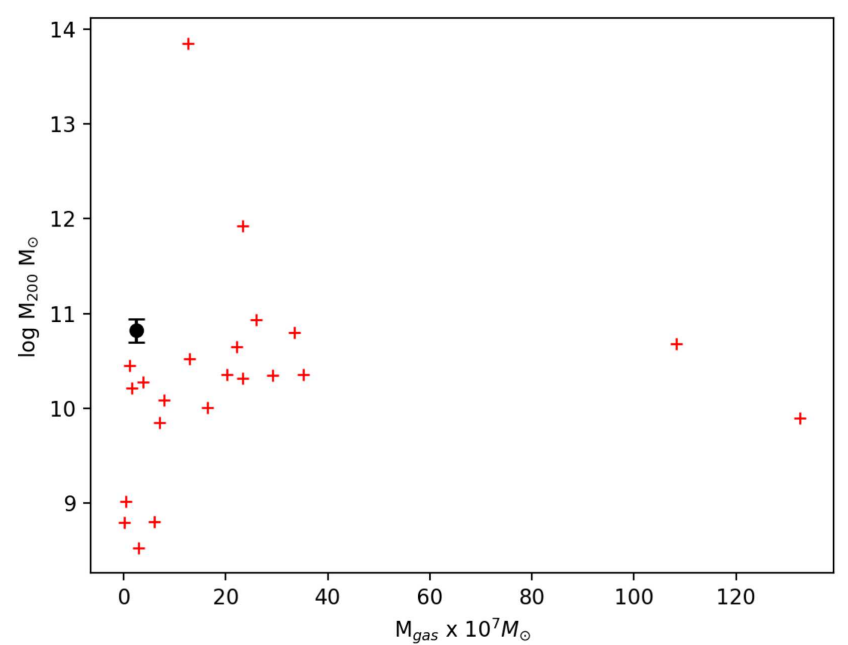

Figure 10. Mean $\mathrm{M}_{200}$ from the two NFW models from section 4.1 for UGC 2162 (black circle) and model fits to LITTLE THINGS dwarf galaxies from Oh et al. (2015) (red crosses) $\mathrm{v} \mathrm{M}_{\text {gas }}$.

for UGC 2162 is a factor 3 higher than the median ratio for the LIITLE THINGs dwarf galaxies of similar $\mathrm{M}_{\text {gas }}$. We plot in Figure 8 the UGC 2162 bayonic and stellar masses vs $\mathrm{V}_{\max }$ compared to both Baryonic and Stellar Tully-Fisher relations from McGaugh et al. (2000); Torres-Flores et al. (2011). For this plot we made the same assumption about the relation of $\mathrm{M}_{g a s}$ to $\mathrm{M}_{H I}$ as we did in Section 4.1, i.e. $\mathrm{M}_{\text {gas }}=1.4 \times \mathrm{M}_{H I}$. The plot shows that while UGC 2162's 
baryonic mass falls above the upper $1 \sigma$ uncertaintly for Baryonic Tully-Fisher relation (BTFR), its stellar mass is within $1 \sigma$ uncertaintly for the Stellar Tully Fisher relation. No strong conclusion should be drawn based on this as the mass estimates for an individual galaxy usually has large uncertainties. But, this result marginally reinforces the indications that UGC 2162 is gas rich relative to its stellar mass, suggesting that some mechanism has in the past suppressed its SF and star formation effiency (SFE) to much lower levels than its currently elevated star formation rate (SFR) of $0.01 \mathrm{M}_{\odot} \mathrm{yr}^{-1}$ estimated by Trujillo et al. (2017). Wong et al. (2016) argues that the secular evolution of a combination of disk stability and disk hydrostatic pressure is the primary driver of the observed $\mathrm{H}_{\mathrm{I}}$ based star formation efficiency $\left(\mathrm{SFE}_{\mathrm{HI}}\right)$. They find angular momentum to have only a secondary effect, concluding that for stable rotating $\mathrm{H}$ I disks the scatter in the $\mathrm{SFE}_{H I}$ from variation in angular momentum is of the order of a factor of $\sim 2$. UGC 2612's SFE $_{H I}$ is a factor of 4 below the average $\mathrm{SFE}_{H I}$ of the Wong et al. (2016) HIPASS-selected sample of SF galaxies. This indicates additional mechanisms may have operated over extended periods (Gyrs) to suppress its $\mathrm{SFE}_{H I}$ or alternatively it recently acquired a substantial fraction of its current $\mathrm{H}_{\mathrm{I}}$ mass.

Although UGC 2162 is part of a loose group of galaxies, it is currently fairly isolated with respect to its nearest physical companion of similar or larger mass (SDSS J023848.50+003114.2) projected $\sim 175 \mathrm{kpc}$ away. The Hı images and kinematics of UGC 2162 show no clear signs of recent major interaction, harassment or environment related effects that could have led to a major gas loss or significantly influence its morphology. On its own the presence of UGC 2162's H I warp does not necessarily indicate a recent interaction as $\mathrm{H}$ I warps are also seen in isolated galaxies which have been free from major tidal interaction for several Gyrs, e.g. (Sengupta et al. 2012; Portas et al. 2011; Scott et al. 2014), where they have been attributed to secular processes and interactions with minor satellites. Based on the projected distance to its nearest neighbour and the relative velocity of $\sim 277 \mathrm{~km} \mathrm{~s}^{-1}$, UGC 2162 could have suffered a tidal interaction $\sim 0.3 \mathrm{Gyr}$ ago with its nearest neighbour $\left(\sim\right.$ less than the galaxy rotation period $\left.{ }^{8}\right)$, which could conceivably be responsible for both the $\mathrm{H}$ I disk warp and currently enhanced SFR. But we argue that if such an interaction did take place, the galaxy's current state implies that it was most likely a minor flyby that did not sufficiently affect UGC 2162's morphology to induce its transformation to an UDG. According to the modelling by (Holwerda et al. 2011), HI morphology asymmetries from major merger interactions remain detectable for between 0.4 to 0.7 Gyrs. So presumably for UGC 2162 an encounter with a similar or larger sized or larger group member would remain obvious in the HI morphology for at least that time. In UGC 2162 we do not see any tidal remnants, tidal features or any gas deficiency, which supports our argument against a recent major interaction. Hence, any process that drove UGC 2162 to become a UDG is most likely a long term internal or secular one. Figure 7 compares the $\mathrm{V}_{\text {rot }}$ of UGC 2162 to those of LITTLE THINGS dwarfs of similar H I mass and we see that UGC 2162 is a slow rotator for its H I mass, in agreement with the Tully-Fisher analysis.

The estimated halo spin parameter $(\lambda)$ using Hernandez et al. (2007)'s estimator for spiral galaxies gives a moderate $\lambda$ value of 0.1 for UGC 2162. Compared to the higher spin UDGs in Leisman et al. (2017) or Spekkens \& Karunakaran (2018),

\footnotetext{
$8 \mathrm{~T}_{\text {rot }}=1.45 \mathrm{Gyr}=2 \pi \mathrm{R}_{H I} / \mathrm{V}_{\text {rot }}$ where $\mathrm{V}_{\text {rot }}=0.5 \Delta \mathrm{W}_{20} / \sin (\mathrm{i}), \mathrm{W}_{20}=50$ $\mathrm{km} \mathrm{s}^{-1}$ and $\mathrm{i}=55^{\circ}$
}

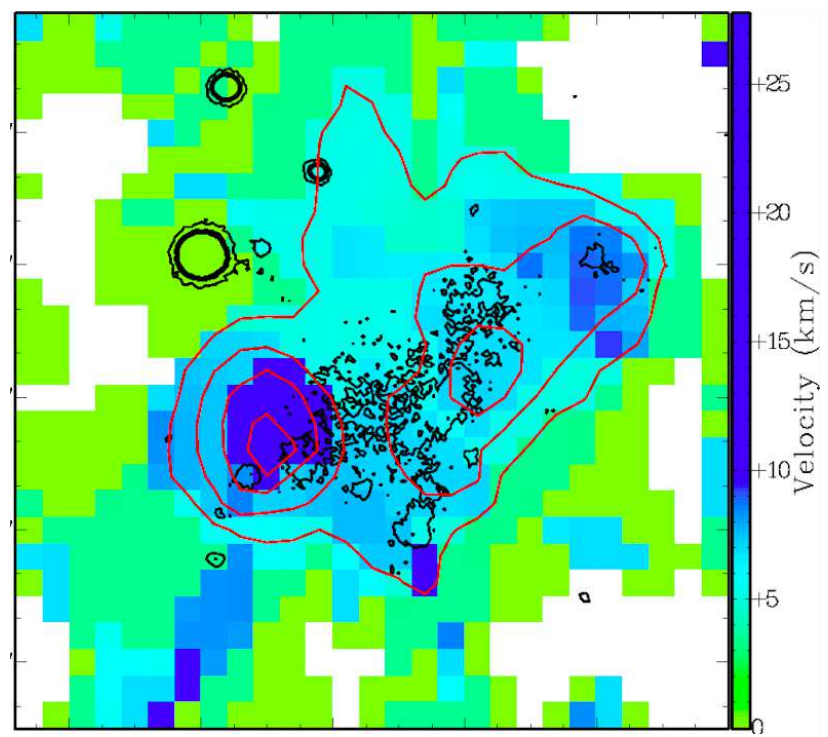

Figure 11. UGC $2162 \mathrm{H}_{\mathrm{I}}$-stellar correlation near optical center: integrated $\mathrm{H}_{\text {I }}$ contours (high resolution, red) and star forming regions (SDSS g-band in black contours) plotted on $\mathrm{H}_{\mathrm{I}}$ velocity dispersion (in blue/cyan) map.

UGC 2162 seems to qualify as a moderate to lower spin galaxy. However, we prefer not to make any claims about halo spin of UGC 2162 as none of the assumptions made to estimate $\lambda$ in Hernandez et al. (2007) and Spekkens \& Karunakaran (2018) matches UGC2162's properties. Instead we prefer to emphasize that most of UGC 2162's properties strongly resemble those of normal dwarf galaxies (e.g. LITTLE THINGS sample) and more importantly the galaxy does not exhibit an abnormally extended disk compared to its DM halo mass to invoke a high spin halo formation scenario for it. From this we infer that for UGC 2162, we can safely rule out the UDG formation scenario involving high spin halos proposed by Amorisco \& Loeb (2016).

The galaxy shows blue star forming regions near the optical centre and the highest UGC $2162 \mathrm{H}_{\text {I }}$ column densities (1.0 $1.3 \times 10^{21} \mathrm{~cm}^{-2}$ ) in the high resolution map approximately coincide with these star forming zones in the SDSS background image (Figure 1). UGC 2162 also has a faint optical disk revealed in the IAC stripe $82 \mathrm{~g}, \mathrm{r}$, i composite image in Figure 1 of Trujillo et al. (2017) of radius $\sim 60$ arcsec with a $\mu_{g}>26$ mag $\operatorname{arcsec}^{-2}$ at the disk edge. Fig 11 shows the highest column density contours (in red) from the high resolution $\mathrm{H}_{\mathrm{I}}$ map overlayed on the high resolution Hi velocity dispersion map. The projected positions of the highest density $\mathrm{H}_{\mathrm{I}}$ and $\mathrm{H}_{\mathrm{I}}$ velocity dispersion maximum in the Figure coincide and are offset $\sim 20 \operatorname{arcsec}(1.2 \mathrm{kpc})$ to the SE of the optical centre. Addionally, there is evidence of diffuse extra-planar $\mathrm{H}_{\mathrm{I}}$ in the pv diagram (Figure 6) coinciding with the highest $\mathrm{H}_{\mathrm{I}}$ dispersion zone. This may indicate some moderate to low level SF related outflow, but the highly disruptive past outflows proposed by van Dokkum et al. (2016) and Di Cintio et al. (2017) to explain the origin of UDGs can be ruled out in this case, given the galaxy is currently undergoing a heightened level of SF and its low accumulated stellar mass. 


\section{SUMMARY AND CONCLUDING REMARKS}

Overall the H I morphology and kinematics of UDG 2162 indicates a rather symmetric $\mathrm{H}_{\mathrm{I}}$ disk within a DM halo with a mass and profile typical of dwarf galaxies. However, the $\mathrm{H}$ i disk contains a warp and a moderate, currently elevated, SFR of $0.01 \mathrm{M}_{\odot}$ which might be attributable to an interaction with a fellow group member in last $\sim 0.3$ Gyr. Comparing UGC 2162 to samples of UDGs with $\mathrm{H}_{\mathrm{I}}$ detections show it to have amongst the smallest $\mathrm{R}_{e}$ with its $\mathrm{MH} \mathrm{I} / \mathrm{M}_{*}$ being higher and $\mathrm{g}-\mathrm{i}$ colour bluer than typical values in those samples. The galaxy is classified as UDG but our investigation could not unambiguously connect its current state to any of the proposed formation scenarios in the literature. On the other hand we could rule out some of the most common proposed scenarios and infer the following: 1) UGC 2162's DM halo is a dwarf halo typical of normal dwarf galaxies 2) the similarity of UGC 2162's properties to those of the LITTLE THINGS sample does not support a scenario requiring abnormally high spin 3 ) the environment and $\mathrm{H}_{\text {I }}$ content of the galaxy does not indicate a formation scenario depending on a recent interaction or environmental assistance; 4) we did not find evidence of recent or past highly disruptive SF driven outflows. A detailed modelling of the galaxy's star formation history may throw more light on how this galaxy formed.

\section{ACKNOWLEDGEMENTS}

We thank the staff of the GMRT who have made these observations possible. The GMRT is operated by the National Centre for Radio Astrophysics of the Tata Institute of Fundamental Research. TS acknowledge support by Fundação para a Ciência e a Tecnologia (FCT) through national funds (UID/FIS/04434/2013), FCT/MCTES through national funds (PIDDAC) by this grant UID/FIS/04434/2019 and by FEDER through COMPETE2020 (POCI-01-0145-FEDER-007672). TS also acknowledges the support by the fellowship SFRH/BPD/103385/2014 funded by FCT (Portugal) and POPH/FSE (EC). TS additionally acknowledges support from DL 57/2016/CP1364/CT0009. This work was supported by FCT/MCTES through national funds (PIDDAC) by this grant PTDC/FIS-AST/29245/2017. Support for this work was provided by the National Research Foundation of Korea to the Center for Galaxy Evolution Research (No. 2010-0027910) and NRF grant No. 2018R1D1A1B07048314. This research has made use of the NASA/IPAC Extragalactic Database (NED) which is operated by the Jet Propulsion Laboratory, California Institute of Technology, under contract with the National Aeronautics and Space Administration. This research has made use of the Sloan Digital Sky Survey (SDSS). Funding for the SDSS and SDSS-II has been provided by the Alfred P. Sloan Foundation, the Participating Institutions, the National Science Foundation, the U.S. Department of Energy, the National Aeronautics and Space Administration, the Japanese Monbukagakusho, the Max Planck Society, and the Higher Education Funding Council for England. The SDSS Web Site is http://www.sdss.org/.This research made use of APLpy, an open-source plotting package for Python hosted at http://aplpy.github.com. This research made use of APLpy, an open-source plotting package for Python (Robitaille \& Bressert 2012). The Parkes telescope is part of the Australia Telescope which is funded by the Commonwealth of Australia for operation as a National Facility managed by CSIRO.

\section{REFERENCES}

Amorisco N. C., Loeb A., 2016, MNRAS, 459, L51

Baars J. W. M., Genzel R., Pauliny-Toth I. I. K., Witzel A., 1977, A\&A, 61,99

Beasley M. A., Trujillo I., 2016, ApJ, 830, 23

Beasley M. A., Romanowsky A. J., Pota V., Navarro I. M., Martinez Delgado D., Neyer F., Deich A. L., 2016, ApJ, 819, L20

Bell E. F., McIntosh D. H., Katz N., Weinberg M. D., 2003, ApJS, 149, 289

Blanton M. R., Hogg D. W., Bahcall N. A., Brinkmann J., Britton M., Connolly A. J., Csabai I., Fukugita M., 2003, ApJ, 592, 819

Broeils A. H., Rhee M.-H., 1997, A\&A, 324, 877

Carleton T., Errani R., Cooper M., Kaplinghat M., Peñarrubia J., Guo Y., 2019, MNRAS, 485, 382

Danieli S., van Dokkum P., Conroy C., Abraham R., Romanowsky A. J., 2019, ApJ, 874, L12

Di Cintio A., Brook C. B., Dutton A. A., Macciò A. V., Obreja A., Dekel A., 2017, MNRAS, 466, L1

Di Teodoro E. M., Fraternali F., 2015, MNRAS, 451, 3021

Espada D., Verdes-Montenegro L., Huchtmeier W. K., Sulentic J., Verley S., Leon S., Sabater J., 2011, A\&A, 532, A117

Haynes M. P., Giovanelli R., 1984, AJ, 89, 758

Hearin A. P., et al., 2017, AJ, 154, 190

Hernandez X., Park C., Cervantes-Sodi B., Choi Y.-Y., 2007, MNRAS, 375,163

Holwerda B. W., Pirzkal N., Cox T. J., de Blok W. J. G., Weniger J., Bouchard A., Blyth S.-L., van der Heyden K. J., 2011, MNRAS, 416, 2426

Koribalski B. S., et al., 2018, MNRAS, 478, 1611

Laporte C. F. P., Agnello A., Navarro J. F., 2019, MNRAS, 484, 245

Leisman L., et al., 2017, ApJ, 842, 133

McGaugh S. S., Schombert J. M., Bothun G. D., de Blok W. J. G., 2000, ApJL, 533, L99

Meyer M. J., et al., 2004, MNRAS, 350, 1195

Oh S.-H., et al., 2015, AJ, 149, 180

Portas A., et al., 2011, ApJL, 739, L27

Robitaille T., Bressert E., 2012, APLpy: Astronomical Plotting Library in Python, Astrophysics Source Code Library (ascl:1208.017)

Román J., Trujillo I., 2017, MNRAS, 468, 4039

Schlafly E. F., Finkbeiner D. P., 2011, ApJ, 737, 103

Scott T. C., et al., 2014, A\&A, 567, A56

Scott T. C., Brinks E., Cortese L., Boselli A., Bravo-Alfaro H., 2018, MNRAS, 475, 4648

Sengupta C., et al., 2012, A\&A, 546, A95

Spekkens K., Karunakaran A., 2018, ApJ, 855, 28

Springob C. M., Haynes M. P., Giovanelli R., Kent B. R., 2005, ApJS, 160,149

Toloba E., et al., 2018, ApJL, 856, L31

Torres-Flores S., Epinat B., Amram P., Plana H., Mendes de Oliveira C., 2011, MNRAS, 416, 1936

Trujillo I., Roman J., Filho M., Sánchez Almeida J., 2017, ApJ, 836, 191

Wang J., et al., 2017, MNRAS, 472, 3029

Wittmann C., et al., 2017, MNRAS, 470, 1512

Wong O. I., Meurer G. R., Zheng Z., Heckman T. M., Thilker D. A., Zwaan M. A., 2016, MNRAS, 460, 1106

Yagi M., Koda J., Komiyama Y., Yamanoi H., 2016, ApJS, 225, 11

Yozin C., Bekki K., 2015, MNRAS, 452, 937

Zaritsky D., 2017, MNRAS, 464, L110

van Dokkum P. G., Abraham R., Merritt A., Zhang J., Geha M., Conroy C., 2015a, ApJL, 798, L45

van Dokkum P. G., et al., 2015b, ApJL, 804, L26

van Dokkum P., et al., 2016, ApJL, 828, L6

van Dokkum P., et al., 2018, ApJL, 856, L30

van der Burg R. F. J., et al., 2017, A\&A, 607, A79 